\title{
HIGHLIGHTS
}

\section{Drugs enter a liquid phase}

cisplatin
partitioning to
condensates
promotes the
activity of
the drug

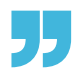

Biomolecular condensates are membraneless subcellular structures, which form via liquid-liquid phase separation. By creating a unique biochemical environment - involving specific concentration and/or exclusion of cellular components biomolecular condensation offers a means to regulate cellular processes as diverse as signalling and gene expression. Changes in condensate properties have also been associated with various human pathologies, including cancer and degenerative diseases. A study from Young and colleagues now shows that chemical compounds can also undergo partitioning into condensates, affecting their activity in cells.

The authors hypothesized that small-molecule drugs could associate with condensates that contain their molecular targets. They focused on anti-cancer compounds and their partitioning into several nuclear condensates that have been associated with gene regulation and RNA processing. These condensates were reconstituted in vitro using individual recombinant proteins, previously shown to phase separate in vitro and to serve as scaffolds for condensate formation.

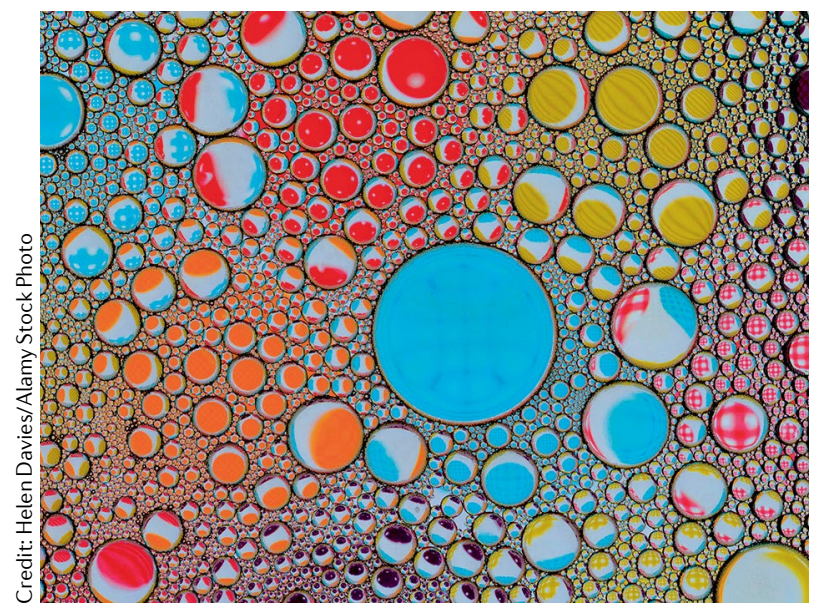

The in vitro reconstituted condensates did not generally accumulate small-molecule compounds, such as fluorescent dyes. However, they could concentrate various anti-cancer drugs, including the DNA-interacting compounds cisplatin and mitoxantrone, as well as tamoxifen, THZ1 and JQ1, which are compounds targeting transcription regulators. This partitioning occurred even in the absence of the drug targets in the condensate. Although different drugs exhibited variable capacity to partition into the condensates, they all showed high enrichment in condensates of the transcription regulator MED1, formation of which has been associated with gene activation by super-enhancers - large clusters of enhancers, which have been associated with oncogenesis.

Incorporation of DNA together with cisplatin into reconstituted MED1 condensates led to robust DNA modification by platination. Consistently, in a colon cancer cell line, platinated DNA frequently co-localized with MED1 condensates, suggesting that cisplatin partitioning to condensates promotes the activity of the drug.

Interestingly, reconstituted MED1 condensates gradually dissolved in the presence of cisplatin, and prolonged treatment of colon cancer cells with cisplatin led to the loss of MED1 from super-enhancers. These data indicate that cisplatin exerts its anti-neoplastic activity by partitioning into super-enhancerassociated biomolecular condensates and causing their dissolution. Because various cancers rely on super-enhancer-mediated oncogene expression, this association of cisplatin with condensates and their subsequent dissolution could explain the efficacy of this general
DNA-modifying compound in cancer therapy.

It has been previously shown by the group that reconstituted MED1 condensates also concentrate the oestrogen receptor. Interestingly, addition of tamoxifen - which is used as an anti-oestrogen compound in cancer therapy - led to eviction of oestrogen receptor from these reconstituted condensates. Notably, tamoxifen resistance is often associated with MED1 overexpression. The authors observed that in tamoxifen-resistant breast cancer cells, the volume of MED1-positive condensates was increased compared with tamoxifen-sensitive cells, and this increase could be recapitulated in vitro by augmenting MED1 concentration. Furthermore, in vitro, the efficacy of tamoxifen in evicting oestrogen receptor was considerably reduced in larger MED1 condensates, which correlated with decreased concentration of tamoxifen in these condensates. These data suggest that condensate enlargement dilutes tamoxifen, thereby reducing its drug activity. Thus, cancer-associated changes in condensate volume can influence the concentration and activity of anti-cancer drugs that partition into them and may represent a mechanism underlying drug resistance.

In summary, small-molecule drugs can partition into biomolecular condensates. This partitioning may represent a mechanism of drug activity, while changes to condensate properties may modulate the therapeutic outcome of condensate-associating compounds. Integration of these findings into drug discovery and screening platforms could improve efficacy of drug development.

Paulina Strzyz

ORIGINAL ARTICLE Klein, A. I. \& Boija, A. et al. Partitioning of cancer therapeutics in nuclear condensates. Science 368, 1386-1392 (2020) RELATED ARTICLE Banani, S. et al. Biomolecula condensates: organizers of cellular biochemistry. Nat. Rev. Mol. Cell Biol. 18, 285-298 (2017) 